\title{
Gaining Ground in Glomerular Diseases
}

\author{
Sharon G. Adler ${ }^{\mathrm{a}}$ Cynthia C. Nast ${ }^{\mathrm{b}}$ \\ a Lundquist Research Institute at Harbor-UCLA Medical Center, David Geffen School of Medicine at UCLA, \\ Torrance, CA, USA; ${ }^{b}$ Department of Pathology, Cedars-Sinai Medical Center, Los Angeles, CA, USA
}

In partnership with Karger Publishers, we proudly launch Glomerular Diseases (GDZ), a first in class, peerreviewed, open access, online journal with a glomerulocentric focus. Our aspirational goal is for this journal to become a central forum for the international dissemination and coordination of cutting edge clinical, basic, and informatics science and practice in glomerular diseases in order to bring breakthrough treatments to patients.

Over the past several decades, glomerular diseases have catapulted to the forefront of a precision medicine revolution in nephrology. There has been an explosion of innovative clinical and discovery science research in this area, with advances in systems biology promising breakthroughs in understanding pathogeneses and providing new therapeutic paradigms. Dedicated investigators around the world are now aligned with large and small pharmaceutical companies to bring new treatments to patients. Foundations representing patients with glomerular diseases have emerged globally, expressing the needs of people with these rare diseases, and directly connecting these patients (and often their parents) to investigators, clinicians, and pharmaceutical developers. Parents, patients, foundations, governmental agencies, pharmaceutical companies, clinicians, and investigators are forging public-private partnerships to advance knowledge, ultimately to bring new treatments to patients. We aim to serve this community in its entirety.

\section{karger@karger.com www.karger.com/gdz

Glomerular disease research publications are currently scattered among many journals otherwise focusing on nephrology, pathology, genetics, precision medicine, biomarkers, health services, patient-related outcomes, and basic science. Glomerular Diseases meets a critical unmet need of the glomerular community by inverting this paradigm, unifying in one focused publication all of these disciplines as they address the glomerulus. We hope that Glomerular Diseases will emerge as the virtual global meeting place for all who have something to contribute to this field, and all who have something to gain.

Glomerular Diseases invites scholarly publications in clinical nephrology; renal pathology; analytic biostatistics and bioinformatics methods and applications; precision medicine; basic discovery science; genetics, genomics, proteomics, and biomarkers; patient-centered research, and continuous quality improvement. The publication will consist of original research, case series, state-of-theart reviews, case reports of unique value, and invited editorial and opinion pieces in these areas. Glomerular diseases are rare (with the exception of diabetic kidney disease), yet patients with these rare diseases comprise at least $20 \%$ of the end-stage kidney disease population in most countries.

Sharon Adler and Cynthia Nast are the founding Editor in Chief and Deputy Editor of Glomerular Diseases. We both have a fundamental commitment to and track- 
record of research and clinical practice dedicated to the study and care of patients with glomerular diseases. Reflective of our training and experience in nephrology and renal pathology, we bring intellectual and personal synergy to the editorial leadership.

Glomerular Diseases boasts an extraordinarily accomplished and passionate group of leading international scholars as Associate Editors and members of its Editorial Board. Karger welcomes as Associate Editors Dr. Alessia Fornoni, Dr. Jonathan Hogan, Dr. Laura Mariani, Dr. Susan Massengill, and Dr. Sydney Tang.

Karger welcomes as Editorial Board members Vinita Agrawal, Rupali Avasare, Arvind Bagga, Ingeborg Bajema, Laura Barisoni, Jonathan Barratt, Moumita Barua, Dawn Caster, Jason Choo, H. Terrence Cook, Tiane Dai, Maria Ferris, Fernando Fervenza, Agnes Fogo, Daniel Gale, Debbie Gipson, Jeffrey Hodgin, Lawrence Holzman, Elion Hoxha, Rachel Jones, Elaine Kamil, Meghan Kapp, Matthias Kreztler, Juan Manuel Mejía, Kana Miyata, Dario Roccatello, Matthew G. Sampson, John Sedor,
Sanjeev Sethi, Geetika Singh, Ben Sprangers, Isaac Stillman, Hitoshi Suzuki, Howard Trachtman, Jonathan Troost, and Jarcy Zee.

Acknowledgement is made to:

- researchers in the field of glomerular diseases who have, with devotion, ingenuity, teamwork, and skill, created a body of engaging and substantive work to concentrate in a single source journal;

- pharmaceutical companies who have ventured into the risky arena of drug development for patients with rare diseases;

- governmental agencies and non-profit foundations who support research in these rare diseases; and

- to patients and their parents, who participate in clinical studies and trials as partners in discovery to find cures.

We hope that Glomerular Diseases, with its global editorial representation and online open accessibility serving an open science philosophy, will grow to become the definitive source for information in this field. 